\title{
ACCOMPLISHMENTS IN BIOASTRONAUTICS RESEARCH ABOARD INTERNATIONAL SPACE STATION
}

\author{
John J. Uri \\ ISS Payloads Office \\ Cynthia P. Haven \\ Bioastronautics Office \\ NASA Johnson Space Center \\ Houston, TX 77058
}

Abstract

The seventh long-duration expedition crew is currently in residence aboard International Space Station (ISS), continuing a permanent human presence in space that began in October 2000. During that time, expedition crews have been operators and subjects for 16 Human Life Sciences investigations, to gain a better understanding of the effects of long-duration space flight on the crewmembers and of the environment in which they live. Investigations have been conducted to study the radiation environment in the station as well as during extravehicular activity (EVA); bone demineralization and muscle deconditioning; changes in neuromuscular reflexes, muscle forces and postflight mobility; causes and possible treatment of postflight orthostatic intolerance; risk of developing kidney stones; changes in pulmonary function caused by long-duration flight as well as EVA; crew and crew-ground interactions; and changes in immune function. The experiment mix has included some conducted in flight aboard ISS as well as several which collected data only pre- and postflight. The conduct of these investigations has been facilitated by the Human Research Facility (HRF). HRF Rack 1 became the first research rack on ISS when it was installed in the US laboratory module Destiny in March 2001. The rack provides a core set of experiment hardware to support investigations, as well as power, data and commanding capability, and stowage. The second HRF rack, to complement the first with additional hardware and stowage capability, will be launched once Shuttle flights resume. Future years will see additional capability to conduct human research on ISS as International Partner modules and facility racks are added to ISS. Crew availability, both as a subject count and time, will remain a major challenge to maximizing the science return from the bioastronautics research program.

\subsection{Introduction}

The International Space Station (ISS) is a unique platform for conducting research in a variety of disciplines, to better understand the role gravity, or its absence, plays in biological and physical processes. As a long-duration laboratory, it enables research that was not possible on earlier platforms. In particular, ISS is ideally suited for studying the effects of long-duration space flight on humans, important for perfecting countermeasures to the deleterious effects to ensure crew safety.

\subsection{ISS Assembly and Operations}

Construction of ISS began in late 1998, with the launch of the Russian module Zarya. Since that time, 33 flights of Russian and American vehicles have added 11 major elements to the station, enlarging the platform from its original 20,000-kg single module configuration to the current $180,000-\mathrm{kg}$ facility depicted in Figure 1a. Russian Soyuz spacecraft and American Space Shuttles have brought seven long duration crews to live aboard the station for 4-6 months each, resulting in a continuous human presence in space since October 2000, and along with unmanned Russian Progress vehicles have brought the required logistics to maintain the station and its crews in a safe and productive environment. Among the resupply items have been seven large research racks and $\mathrm{xx} \mathrm{kg}$ of research hardware logistics.

A significant milestone for ISS-based research was the addition of the US Laboratory Module Destiny, launched on the STS 98/5A mission in February 2001. Weighing 14,000 kg at launch, Destiny is a cylindrical module, $8.5 \mathrm{~m}$ long with an external diameter of $4.3 \mathrm{~m}$. Internally, it is configured with 24 racks lining the four surfaces. The racks contain various systems equipment such as life support, controls 
for the station's robotic arms, medical hardware, a crew sleep station, and up to 10 research facilities, of which seven are already on orbit. The first research rack, to be described in more detail below, was the Human Research Facility (HRF) Rack 1, installed a month after Destiny's arrival.

(a)

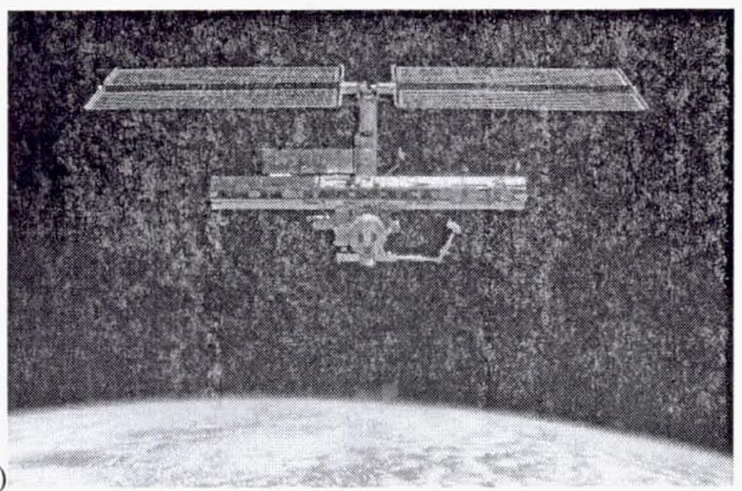

(b)

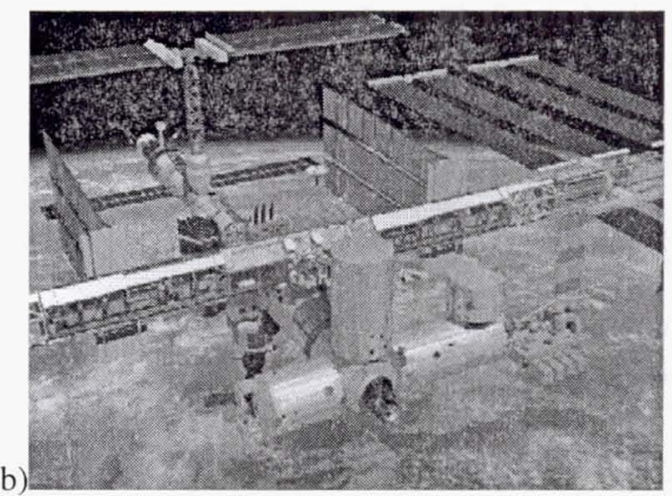

Figure 1. (a) Current configuration of ISS; (b) configuration of ISS including International Partner modules.

Assembly of ISS will continue in the near future with completion of the trusses, addition of solar arrays for power generation and radiator panels for cooling, and the International Partner research modules, leading to a configuration represented in Figure 1b. The European Space Agency's Columbus module will increase the station's research capability with an additional 10 research rack locations, with the Japanese Kibo adding 11 more. The full suite of research modules will also allow optimal location of life sciences research racks for maximal synergy.

Concurrent with the launch of the first research rack in March 2001, the Payload Operations and Integration Center (POIC) at the Marshall Space Flight Center (MSFC) in Huntsville, AL, became operational. Additional payload-specific support beginning with Expedition 2 was provided by Telescience Support Centers other NASA Centers, including Johnson Space Center for human life sciences investigations. Remote sites at investigators' institutes, domestic and international, are also routinely tied in during times when those experiments are operating. The Telescience Resource Kit (TReK) developed by the MSFC proved to be a very reliable tool providing remote payload developers the ability to monitor and control their payloads from their home locations.

\subsection{Facilities for human research}

The two HRF Racks (Figure 2) provide a core set of experiment hardware to support science investigations, as well as power, command and data handling and stowage, described below. The first rack has been on orbit since March 2001, while the second will be launched on the first mission after Shuttles resume flying. These racks will initially reside in Destiny, but will be relocated to Columbus once that module arrives, where they will be joined by the European Physiology Module (EPM) and the Muscle Atrophy Research and Exercise System (MARES) racks. The EPM rack will provide the infrastructure for up to eight Science Modules in a variety of life science research disciplines, while the MARES facility is capable of assessing strength of muscle groups around a single joint or complete limb. These combined facilities, along with cold stowage capability provided by the ESA-provided Minus Eighty-degree Laboratory Freezer for ISS (MELFI), which can store materials at $-80^{\circ} \mathrm{C},-22^{\circ} \mathrm{C}$ and $+4^{\circ} \mathrm{C}$, will establish a powerful suite of instruments for conducting in-depth studies of human physiology in microgravity.

Integral to the functioning of the HRF Racks are the computer Workstations and laptops. Each rack has its own Workstation, with the Rack 2 Workstation containing enhanced technologies allowed by its later launch. The Workstations provide for data collection and storage, software interfaces to various experiments, data downlinking, video processing and graphics support. The laptop provides a platform for installing and executing software to perform various rack functions as well as experiment procedures. 


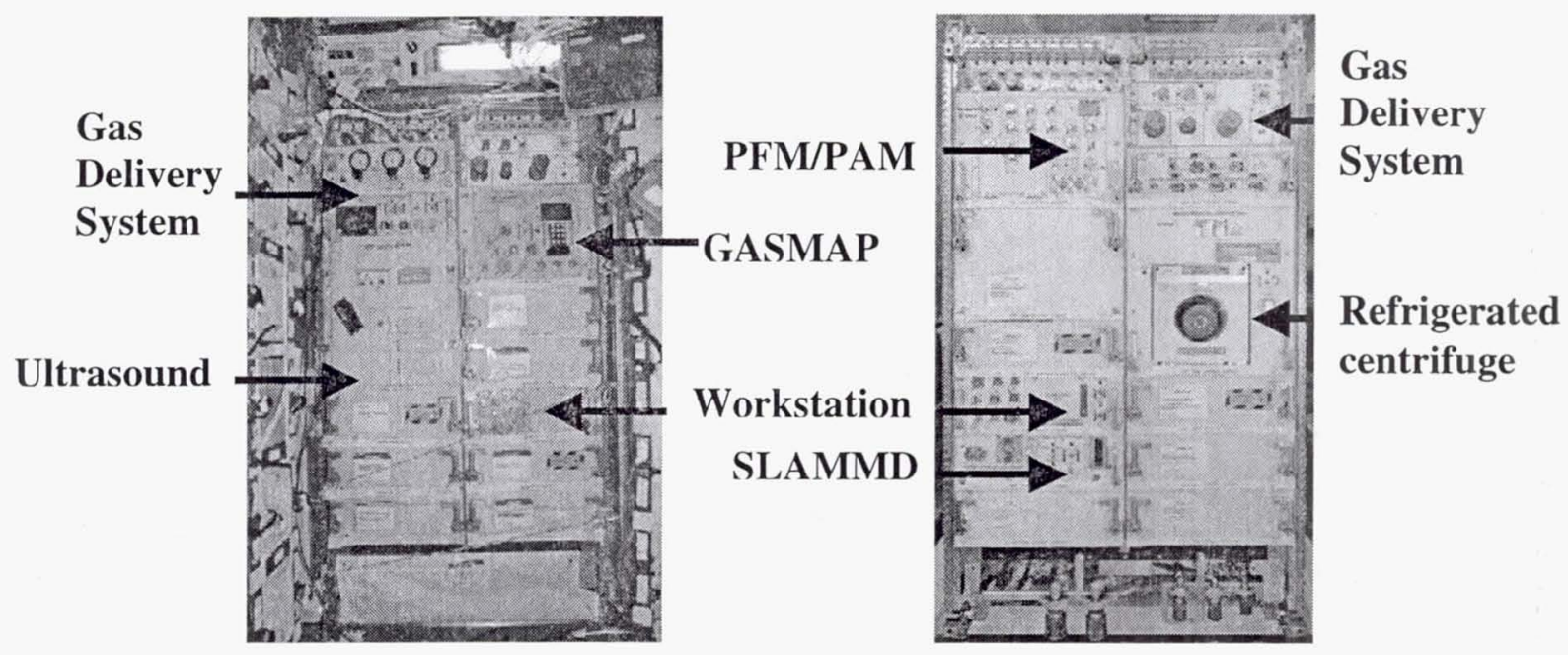

Figure 2. Human Research Facility Racks 1 and 2. Subcomponents described in text.

The Pulmonary Function System (PFS) is a collaborative effort between the United States and ESA to develop a system for pulmonary physiology monitoring. The system will be complete once both HRF racks are on orbit. The components of the system are: Pulmonary Function Module (PFM), provided by ESA; Photoacoustic Analyzer Module (PAM), provided by ESA; Gas Analyzer System for Metabolic Analysis Physiology (GASMAP), provided by NASA; and Gas Delivery System (GDS), provided by NASA. The PFM plus GDS can operate together with either GASMAP or PAM, in the latter case the system is portable instead of rack-based as in the former case. The system can provide a wide range of respiratory and cardiovascular measurements, including breath-by-breath measurements, pulmonary volumes and capacities, spirometry, cardiac output and other specialized tests of pulmonary function.

The Ultrasound device located in HRF Rack 1 provides for multiple imaging modes including Doppler for a variety of applications, both for science investigations as well as operational medical diagnostics. The upgradable system can both record images locally and downlink imagery. In addition to 2D imaging, it can also provide post-image processing to yield $3 \mathrm{D}$ reconstructions.

Of importance to many biomedical investigations as well as to medical operations is the ability to accurately measure crewmember body mass. The Space Linear Acceleration Mass Measurement Device (SLAMMD) follows Newton's Second Law of Motion by having two springs generate a known force against a mounted crewmember, the resulting acceleration being used to calculate the subject's mass. The device is accurate to $0.25 \mathrm{~kg}$ over a range from $40 \mathrm{~kg}$ to $115 \mathrm{~kg}$.

Collection and in situ processing, such as density-based separation, of fluid samples is integral to many biomedical experiments and to flight medicine. The 6-chamber rotor in the Refrigerated Centrifuge can accommodate sample sizes from $0.5 \mathrm{ml}$ to $50 \mathrm{ml}$, with speeds ranging from 1000 to $5000 \mathrm{rpm}$. The rotor chamber is maintained at $+4^{\circ} \mathrm{C}$.

Integral to most human life sciences investigations is the collection of preflight and postflight data. Facilities for ground-based data collection are established at Johnson Space Center in Texas where astronauts train, at Kennedy Space Center in Florida, the launch site and primary landing site for Space Shuttles, and Dryden Flight Research Center in California, the alternate Shuttle landing site. In the aftermath of the Columbia accident, Russian Soyuz vehicles temporarily replaced the Shuttle as the crew transport vehicle. Therefore, data collection facilities were established on short notice at Gagarin Cosmonaut Training Center outside of Moscow to enable US investigations.

4.0 Investigations 